\title{
FEM Analysis of the Transformer Insulation XY Model
}

\author{
J. Cheng, P. Werelius and M. Ohlen
}

\begin{abstract}
Dielectric Frequency Response, DFR (also known as Frequency Domain Spectroscopy, FDS), was introduced more than 20 years and has been thoroughly evaluated and proven in a number of research projects as and field tests with good results. DFR data in combination with mathematical modeling of the oilpaper insulation is proven as an excellent tool for understanding insulation properties e.g. moisture content in cellulose insulation and temperature dependence of the insulation system.

The traditional XY model is widely known for describing the dielectric response of the paper-oil insulation system. This paper explores the accuracy and limitation of the $X Y$ model by comparing it with FEM using COMSOL Multiphysics.

The 'hump' phenomenon that sometimes is seen in field measurement and cannot be described by the simple XY-model is also investigated by using the FEM technique. The analysis shows that the deviation in the frequency response can be described and modeled as a semi-conductive layer with a certain conductivity and thickness.
\end{abstract}

Index Terms - Cellulose, dielectric response, DFR, FDS, FEM, insulation, modeling, power transformer, semi-conductive layer

\section{INTRODUCTION}

$\mathrm{D}$

FR measurements on transformers and bushings in combination with insulation modeling is a good tool for investigating and understanding the properties of an insulating material. The model used to model the insulation system inside a transformer is usually called and described as an XY-model. The insulation structure is represented by the relative amount of spacers (sticks) and barriers in the cooling duct as shown in Fig. 1. Parameter $\mathrm{X}$, is defined as the ratio of the sum of all barriers in the duct, lumped together, and divided by the duct width. The spacer coverage $\mathrm{Y}$, is defined as the total width of all the spacers divided by the total length of the periphery of the duct.

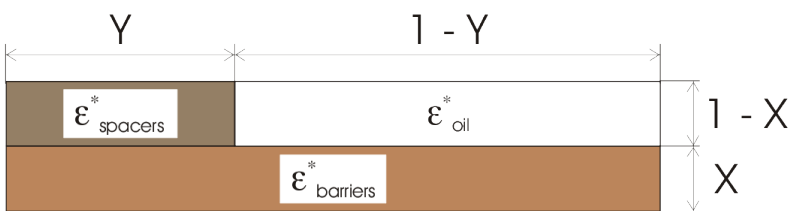

Fig. 1 Sketch diagram of the XY model

The permittivities of oil, $\varepsilon^{*}$ oil , spacers, $\varepsilon^{*}{ }_{\text {spacers }}$ and barriers, $\varepsilon^{*}$ barriers, are complex functions of both frequency and temperature dependent.

\section{Permittivity OF THE XY-MODEL}

The calculation of the total capacitance of the equivalent model can be calculated by various methods. The most rigorous method is solving the 2-dimensional Laplace equation. However, the boundary condition makes the solution extremely complicated so that it is meaningless to apply it in practical situation. The widely used capacitor model is discussed and finite element method (FEM) is used for comparison.

\section{A. Capacitor model}

The total dielectric response of the XY model can be calculated by four capacitors in series and parallel with each other. Dividing the geometry capacitor, the total permittivity could be obtained. The widely applied method is that the capacitors are in series in $\mathrm{X}$ direction and in parallel in $\mathrm{Y}$ direction.

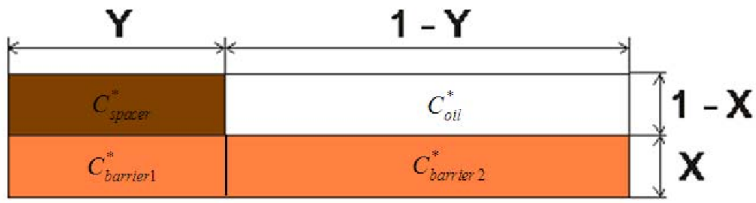

Fig. 2 Capacitor model of the XY model

$$
\varepsilon(\omega, T)_{1}=\frac{Y}{\frac{1-X}{\varepsilon_{\text {spacer }}}+\frac{X}{\varepsilon_{\text {barrier } 1}}}+\frac{1-Y}{\frac{1-X}{\varepsilon_{\text {oil }}}+\frac{X}{\varepsilon_{\text {barrier } 2}}}
$$

\section{B. Finite element method}

The main problem of the capacitor model is that it neglects the electric field distortion due to the discontinuous effect. Fig. 3 is the simulation of voltage distribution in the oil-paper system at $0.01 \mathrm{~Hz}$ in COMSOL. The distorted equal potential lines show that it is not wise to neglect the discontinuous effect. 


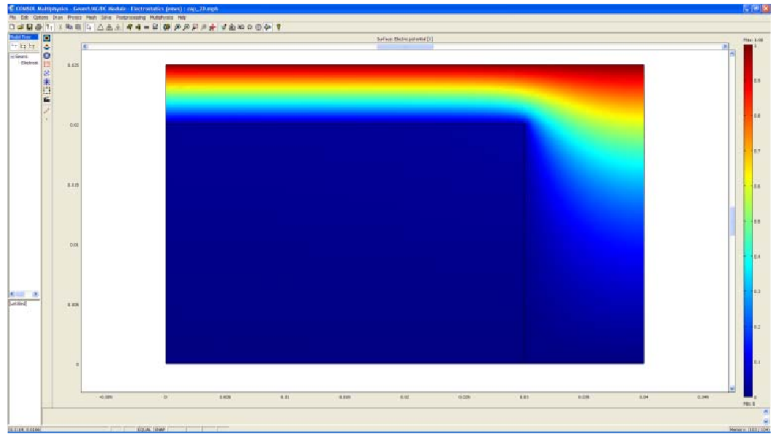

Fig. 3 Simulation of the oil-paper system at $0.01 \mathrm{~Hz}$

The relative error of the measured permittivity between the capacitor model (CM) and COMSOL is defined as:

$$
\text { Error }=(\text { COMSOL }-\mathrm{CM}) / C O M S O L
$$

Results of simulations show that the error varies with geometry, relative permittivies of the two materials and the oil conductivity. Also the error magnitude varies with frequency.

Example of simulation using two different geometries is shown in Fig. 4 and Fig. 5; eprim is real part of permittivity, bis is complex part of permittivity and tand the dissipation factor $=$ bis/eprim.

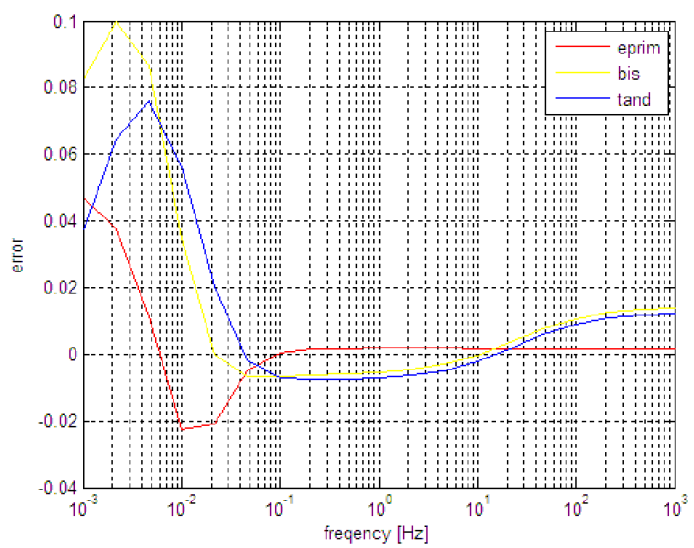

Fig.4 The relative errors with $\mathrm{X}=20 \%$ and $\mathrm{Y}=20 \%$, the oil conductivity sigma $=1 \mathrm{e}-11 \mathrm{~S} / \mathrm{m}$ and the moisture content of the pressboard is $1 \%$.

From the figures, it is obvious that the simplified capacitor model causes errors, especially at low frequencies.

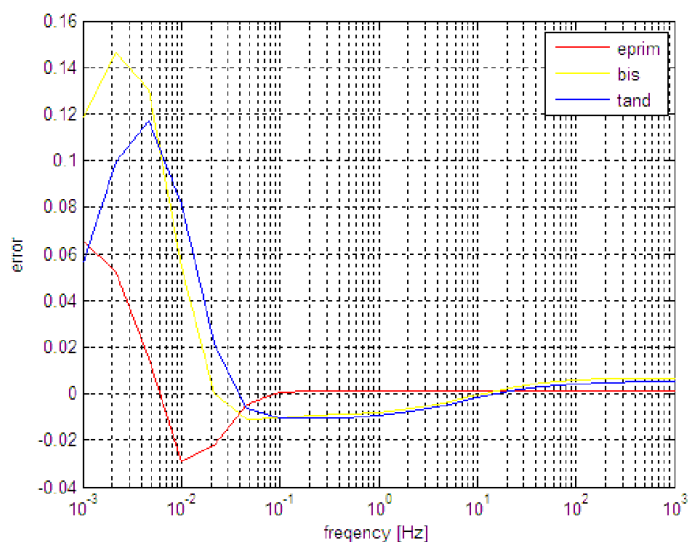

Fig. 5 The relative errors with $\mathrm{X}=20 \%$ and $\mathrm{Y}=50 \%$, the oil conductivity sigma $=1 \mathrm{e}-11 \mathrm{~S} / \mathrm{m}$ and the moisture content of the pressboard is $1 \%$.

\section{The modified capacitor model}

Since the analytical solution of the $\mathrm{XY}$ model is not available, some other method should be used to get the more accurate equivalent permittivity. In widely applied capacitor model, the capacitors are in series in $X$ direction and parallel in $\mathrm{Y}$ direction. But the capacitors also can be in parallel in $\mathrm{Y}$ direction and in series in $\mathrm{X}$ direction, which is given by:

$$
\varepsilon(\omega, T)_{2}=\frac{1}{\frac{X}{Y \varepsilon_{\text {sticks }}+(1-Y) \varepsilon_{\text {barrier }}}+\frac{1-X}{Y \varepsilon_{\text {spacer }}+(1-Y) \varepsilon_{\text {oil }}}}
$$

The true value lies between two permittivities described by equation 1 and equation 3 and can be expressed by a weigh factor $\mathrm{K}$ :

$$
\varepsilon_{e q}=(1-K) \varepsilon_{1}+K \varepsilon_{2}
$$

By choose the proper $\mathrm{K}$, the relative error to COMSOL is shown in Fig.6. The error is greatly reduced compared with Fig.4. 


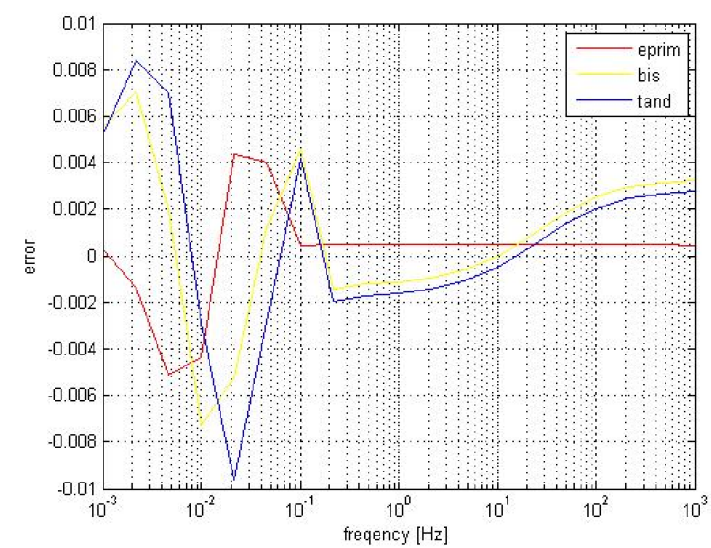

Fig.6 The relative errors of the modified capacitor model with $\mathrm{X}=20 \%$ and $\mathrm{Y}=20 \%$, the oil conductivity sigma $=1 \mathrm{e}-11$ and the moisture content of the pressboard is $1 \% . \mathrm{K}=0.3$ at frequencies below $0.1 \mathrm{~Hz}$ and $\mathrm{K}=0.12$ at frequencies above $0.1 \mathrm{~Hz}$

\section{CONTAMINATION PHENOMENON}

Sometimes the dielectric response of the transformer insulation looks "abnormal" and cannot be related to and described by the simple XY model. An example is shown in Fig.7. It is believed that the "hump" is related to some form of contamination of the insulation material in the transformer.

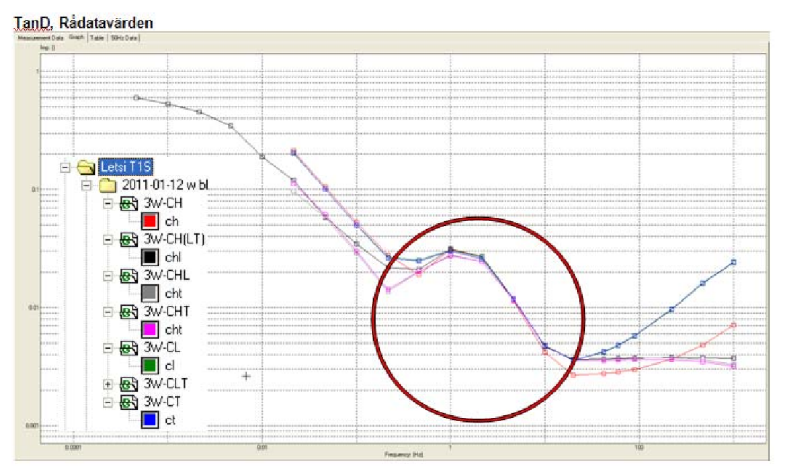

Fig.7 Transformer insulation with unusual deviation of the frequency response ("hump")

\section{A. Physical model of the contamination layer}

The electric field in a two-plate homogeneous capacitor is perpendicular to the electrodes. However, there is also an electric field parallel to the electrodes in the discontinuous oilpaper system as shown in Fig. 8 (a). If the conductive contaminants are accumulated on the surface of the pressboard, the R-C circuit is formed which is shown in Fig.8 (b).

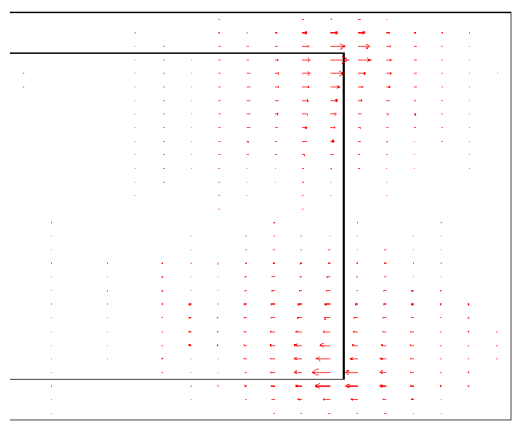

(a) Electric field in parallel with the electrodes V

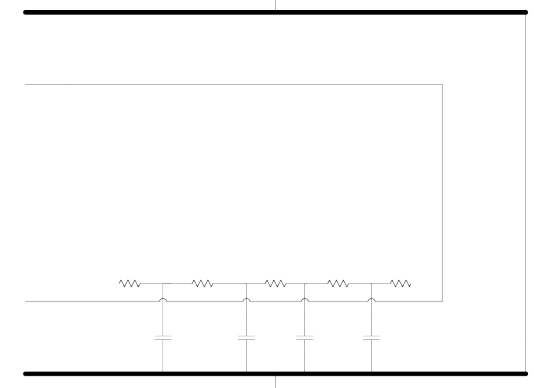

(b) Equivalent R-C circuit

Fig. 8 The effect of the contamination layer in the discontinuous oil-paper insulation system

\section{B. Simulation of the contamination layer in COMSOL}

The contamination layer is added to the interface of oil and cellulose. The thickness and conductivity of the contamination layer are varied respectively.

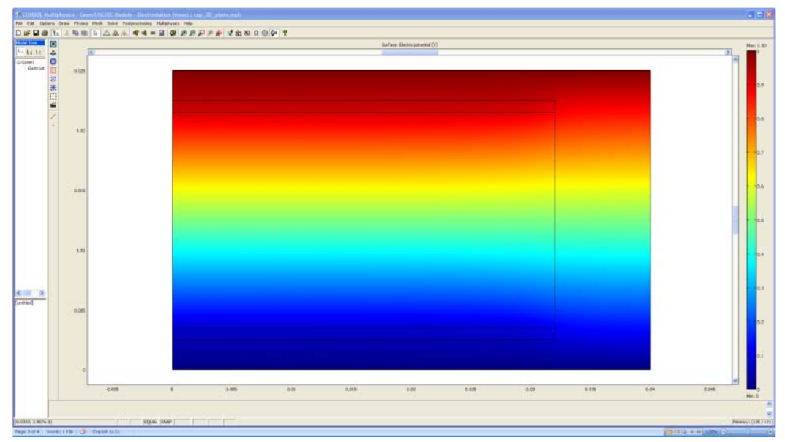

Fig. 9 The contaminated oil-paper system in COMSOL

The dielectric responses with different conductivities are shown in Fig. 10. The contamination layer adds a loss peak to the total response. The conductivity determines the loss peak position, i.e. where in the frequency range the "hump" appears. 

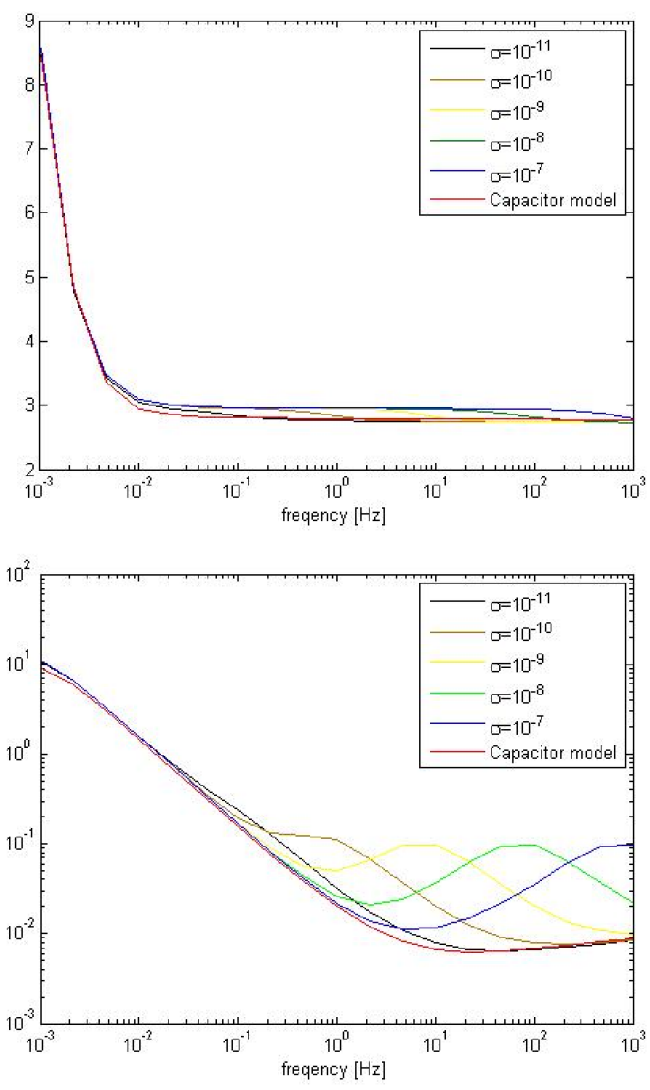

Fig. 10 Dielectric response of the oil-paper system with contamination layers having different conductivities, $\sigma$ in $\mathrm{S} / \mathrm{m}$. The upper figure is the relative real part of the complex permittivity while the lower part is the imaginary part.

The dielectric responses with different layer thicknesses are shown in Fig. 11. The thickness only contributes to the magnitude of the Debye function while the loss peak frequency is not affected.

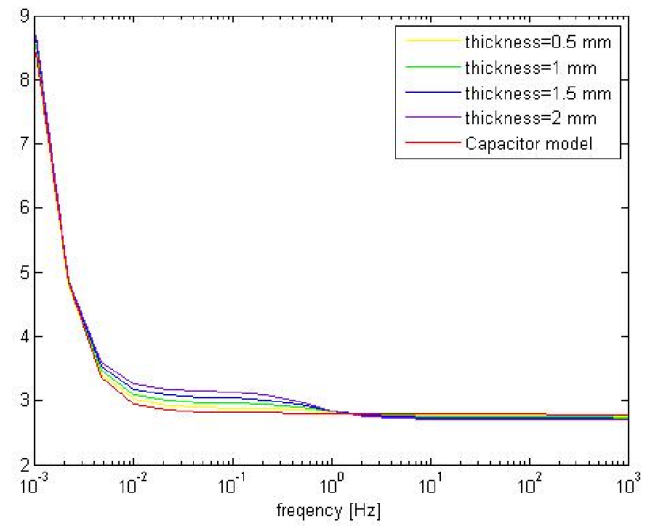

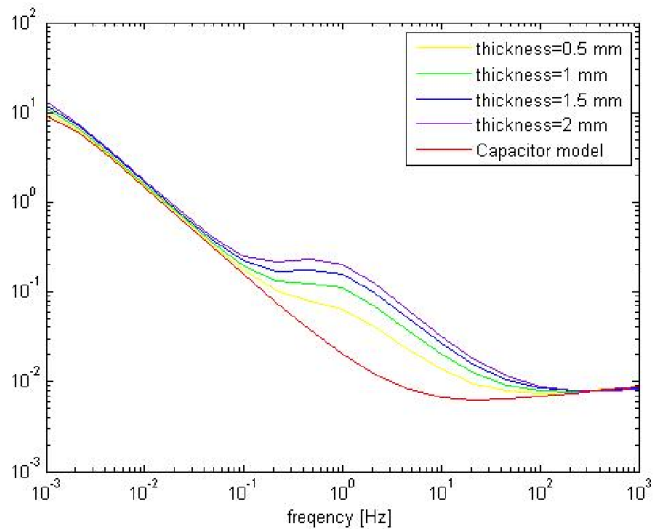

Fig. 11 Dielectric response of the oil-paper system with contamination lavers having different thicknesses. The upper figure is the relative real part of the complex permittivity while the lower part is the imaginary part.

The FEM simulations show that the effect of contamination can be added to the original XY-response in terms of a simple $\mathrm{RC}$-circuit or a bit more advanced model giving a more flat hump. In figure 12 and example of such a modeled hip is shown.

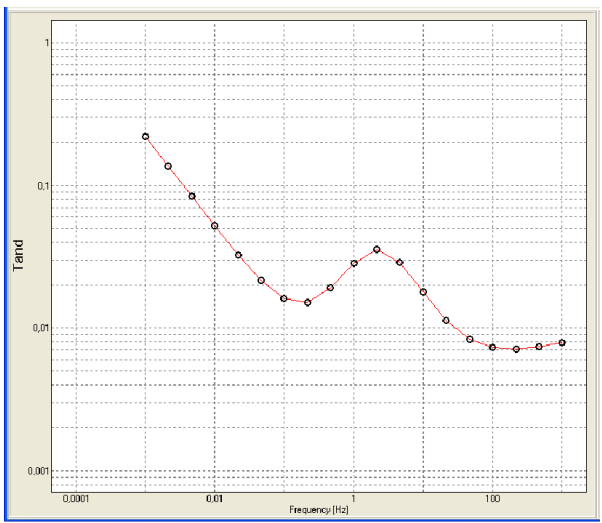

Fig. 12 A Dielectric response curve with a hump modeled using the traditional $\mathrm{XY}$ model with an added hump/peak simulating e.g. a contamination layer.

\section{CONCLUSIONS}

The $\mathrm{XY}$ model is a simple model describing the ratio of the solid and liquid insulation in a power transformer. FEM simulation has been used to simulate errors generated by describe the $\mathrm{XY}$ model in terms of parallel capacitors, and an improved model is proposed.

Using FEM simulations, it is also understood how contamination layers influence the dielectric response. This make it possible understand how peaks/humps in the dielectric response function may be generated by contamination layers.

Finally, the simulations show that a contamination layer pretty simple can be modeled by adding a loss model in parallel with 
the original XY model.

\section{REFERENCES}

[1] P. Werelius et al, "Diagnosis of Medium Voltage XLPE Cables by High Voltage Dielectric Spectroscopy", paper presented at ICSD 1998.

[2] U. Gäfvert, G. Frimpong and J. Fuhr, "Modelling of Dielectric Measurements on Power Transformers', Paper 15-103, CIGRÉ Session Paris 1998.

[3] U. Gäfvert, L. Adeen, M. Tapper, P. Ghasemi, B. Jönsson, "Dielectric Spectroscopy in Time and Frequency Domain Applied to Diagnostics of Power Transformers", Proc. Of the 6th ICPADM, Xi'an, China, 2000

[4] P. Werelius, M. Ohlen, "Using Dielectric Frequency Response Measurements to Determine Dissipation Factor Temperature Dependence in Power System Components", GCC Power 2010, Doha, Qatar

\section{BIOGRAPHIES}

Jialu Cheng was born in Su Zhou, China, 1987. He received his bachelor's degree in Southeast University, China. He graduated from the Royal Institute of Technology (M.Sc. EE) in 2011. His master thesis focused on the broadband dielectric response of paper insulation from $1 \mathrm{mHz}$ to $3 \mathrm{GHz}$. He started work in Megger from 2011 as a research engineer.

Peter Werelius was born in Stockholm, Sweden, 1966. He has

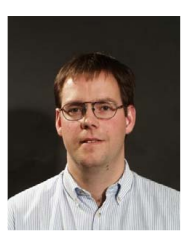

a Master of Science in Electrical Engineering (1991) and a Doctorate Electrical Engineering (2001) both at KTH (Royal Institute of Technology) in Stockholm, Sweden. He started his professional career starting up a spin-off company, WaBtech, in 1996, manufacturing FDS/DFR (Frequency Domain Spectroscopy/Dielectric Frequency Response) test equipment for manly applied to cables and power transformers. From 1999, he continued the work on the FDS/DFR application, now within Programma Electric and later within GE Energy Services after GE acquired Programma in 2002. In 2005, he together with others founded Pax Diagnostics. He was chairman of the board and his primary responsibilities were application and product management. In 2008 Pax Diagnostics was acquired by Megger and Peter is now working as product manager and technical specialist in transformer testing. Peter Werelius has published a number of papers/articles mainly related to FDS/DFR measurement techniques and application. He is member of IEEE and Cigré and takes active part in work groups and task forces.

Matz Ohlen was born in Sundsvall, Sweden, 1952. He

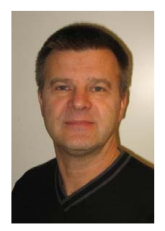
graduated from the Royal Institute of Technology (M.Sc. EE) in Sweden in 1975 and started his professional career at IFM Akustikbyran AB, a consultant company in signal analysis, acoustics and noise \& vibration control. He continued working in industrial automation and measurement technique at Karlson Instruments and Programma Electric, specializing in instrumentation for diagnostic testing of power industry components. In 2000 he started to work in the semiconductor business as Product Manager for Bluetooth products at National Semiconductor. In 2007 he returned to the power industry business as Marketing and Sales Manager for Pax Diagnostics, specializing in test systems for SFRA (Sweep Frequency Response Analysis) and FDS/DFR ((Frequency Domain Spectroscopy/Dielectric Frequency Response Measurements) in transformers, generators and cables. In October 2008 Pax Diagnostics was acquired by Megger and he is now working as Director - Transformer Test Systems. Matz Ohlen has presented a number of papers/articles on Measurement Techniques, Signal Analysis, Wireless Communications, Test and Maintenance of Power System Components etc, for international conferences/publications. $\mathrm{He}$ is member of IEEE. 